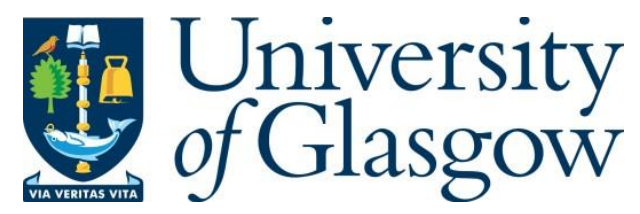

Gamba-Sanchez, D. and Prunet, J. (2018) Synthesis of 1,3-diols by O-nucleophile additions to activated alkenes. Synthesis, 50(20), pp. 3997-4007.

There may be differences between this version and the published version. You are advised to consult the publisher's version if you wish to cite from it.

http://eprints.gla.ac.uk/167718/

Deposited on: 27 August 2018

Enlighten - Research publications by members of the University of Glasgow http://eprints.gla.ac.uk 


\section{Synthesis of 1,3-diols by 0 -nucleophile additions to activated alkenes}

\author{
Diego Gamba-Sánchez ${ }^{\mathrm{a}}$ \\ Joëlle Prunet ${ }^{b}$ \\ ${ }^{a}$ Laboratory of Organic Synthesis, Bio and Organocatalysis, \\ Chemistry Department, Universidad de los Andes. Cra 1 No. \\ 18A-12 Q:305. Bogotá 111711, Colombia. \\ ${ }^{b}$ WestCHEM, School of Chemistry, University of Glasgow, \\ Joseph Black Building, University Avenue, Glasgow G12 8QQ, \\ UK. \\ da.gamba1361@uniandes.edu.co \\ joelle.prunet@glasgow.ac.uk
}

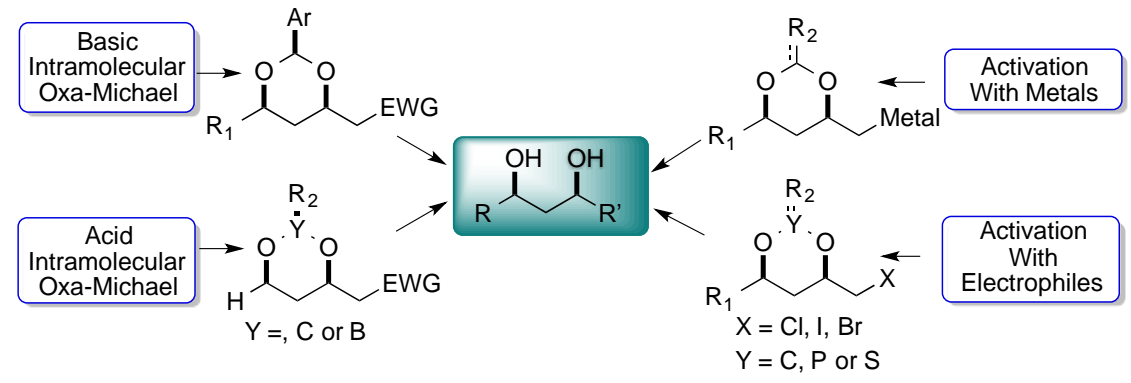

Abstract The diastereoselective synthesis of 1,3-diols by addition of oxygen nucleophiles to activated alkenes is presented. This review focuses on homoallylic alcohol substrates that react with a relay compound to form an intermediate oxygen nucleophile, which in turn will lead to a protected 1,3diol by intramolecular addition to the olefin moiety.

1. Introduction

2. Base Catalysis

3. Organocatalysis

4. Activation with non-metallic electrophiles

5. Activation with transition metal derivatives

6. Conclusions

Key words 1,3-Diols; oxa-Michael; oxygen nucleophiles; activated alkenes; Tsuji-Trost reaction.

\section{Introduction}

The polyol and more specifically the 1,3-diol motifs are ubiquitous in polyketide natural products, whether as free alcohols or masked as cyclic hemiacetals, tetrahydrofuran and tetrahydropyran rings. The stereoselective synthesis of 1,3-diols is a vast topic that was reviewed by Müller et al. in 2006. ${ }^{1} \mathrm{~A}$ comprehensive update of this review focused on transitionmetal catalyzed reactions was recently reported by Kumar and coworkers. $^{2}$

Herein, we want to present an overview of the synthesis of 1,3diols from homoallyllic alcohols where the alcohol function attacks a relay compound to form an intermediate bearing an oxygen nucleophile, which will further attack an activated alkene in an intramolecular fashion (Scheme 1).

The alkene can be activated by the $\mathrm{R}_{3}$ group, e.g. substrates with electron-withdrawing groups will undergo oxa-Michael additions, $^{3}$ or by an external electrophilic agent. This review is divided into four chapters, according to the activation process: base catalysis, organocatalysis, activation with non-metallic electrophiles and activation with transition metal derivatives.

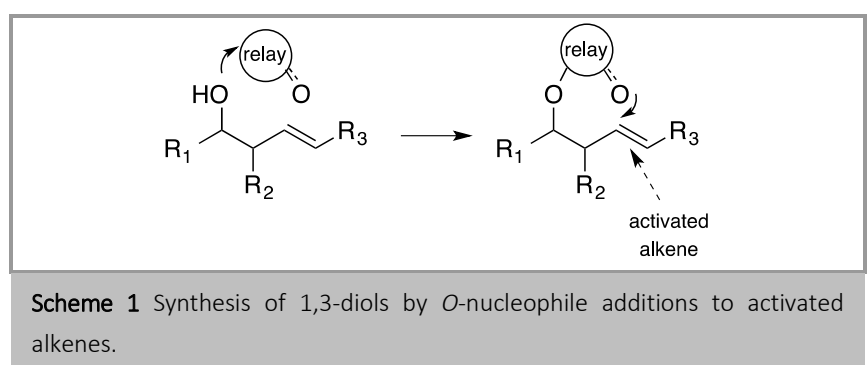

\section{Base Catalysis}

In 1993, Evans and Prunet reported a seminal reaction for the synthesis of protected syn 1,3-diols. ${ }^{4}$ Inspired by the formation of tetrahydropyrans by intramolecular addition of alkoxides to conjugated enones, ${ }^{5}$ they designed a system where benzaldehyde would serve as a relay between the alkoxide and the Michael acceptor to establish a 1,3 relationship between two protected alcohols (Scheme 2). They established that the reaction is under thermodynamic control, giving as the major product the dioxane isomer with all equatorial substituents. In order to drive the reaction to completion, three successive additions of excess benzaldehyde and a catalytic amount of base are needed. Both conjugated esters and Weinreb amides are suitable substrates, but not conjugated ketones or aldehydes; however, the corresponding products can easily be accessed from the Weinreb amide derivatives. Substitution at the allylic position is tolerated, but the diastereoselectivity slightly drops when the alcohol in a syn relationship with this substituent, which occupies an axial position in the major diastereomer. Other aldehydes were investigated; with $p$-anisaldehyde, the reaction is sluggish and with $p$-nitrobenzaldehyde, the reaction is plagued by numerous side-products. The use of aliphatic aldehydes leads to 1:1 mixtures of syn and anti protected diols. This reaction has been employed in the synthesis of numerous natural products. ${ }^{6}$ 


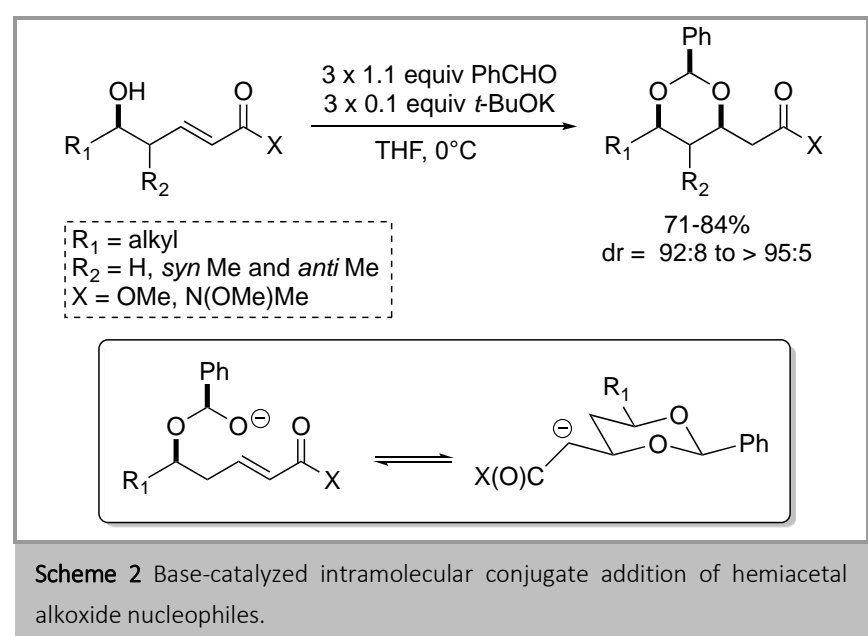

Other Michael acceptors were explored for this reaction. Sulfones were chosen by Prunet et al. as a handle to further functionalize the protected 1,3 diol products with an $\alpha$-olefin, in order to construct a motif found in polyketide natural products such as Dolabelides. ${ }^{7}$ The reaction was successful with a range of aliphatic substrates, and the products were easily purified by recrystallization (Scheme 3). However, further transformation of the sulfones into the desired olefins by Julia olefination proved problematic. ${ }^{8}$

Scheme 3 Oxa-Michael addition to conjugated sulfones.

To circumvent this problem, the same group devised a cascade oxa-Michael/elimination sequence. ${ }^{9}$ Condensation with the required aldehyde or ketone for the Julia olefination was performed on the vinyl sulfone, before the conjugate addition reaction, and the resulting alcohol was transformed into a good leaving group (OLG in Scheme 4). Treatment of these compounds with excess benzaldehyde and a full equivalent of base led to the benzylidene acetals flanked by a vinyl sulfone moiety. The good syn selectivity proves that the thermodynamic equilibrium of the conjugate addition is reached before the elimination occurs. The $E / Z$ mixture of vinyl sulfone isomers is inconsequential, as reduction of the sulfone is stereoconvergent and leads to the desired olefins with excellent $E$ selectivity.

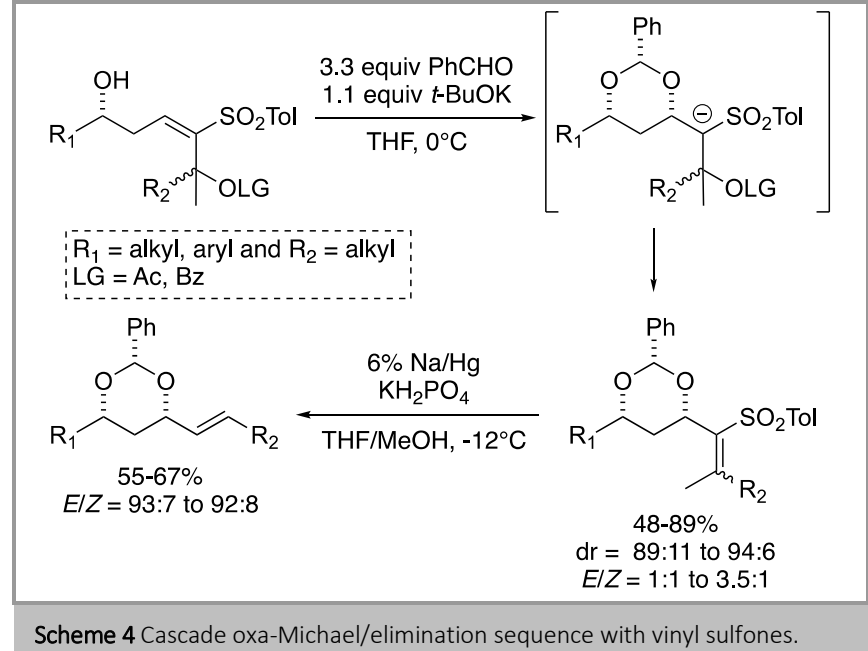

A similar cascade was performed with conjugated esters, and the Morita-Baylis-Hillman adducts were obtained in moderate to good yields and excellent selectivities (Scheme 5).10 If the reaction was performed at $0^{\circ} \mathrm{C}$, the selectivity was only 80:20, but lowering the temperature slowed down the elimination reaction while the equilibration to the more stable isomer was still taking place, leading to a better syn/anti ratio.

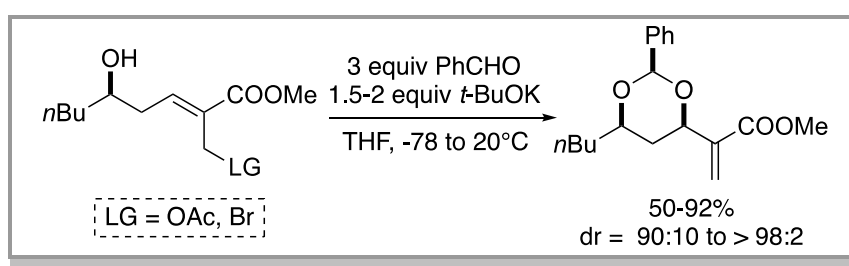

Scheme 5 Cascade oxa-Michael/elimination sequence with conjugated esters.

The modified Julia olefination reaction ${ }^{11}$ employs heteroaromatic sulfones, and gives the olefinic product in single step. In an attempt to optimize the synthesis of protected syn 1,3-diols with an $\alpha$-olefin, Prunet and Oriez developed the oxaMichael reaction on heteroaryl vinyl sulfones. ${ }^{12}$ A careful selection of the reaction conditions is needed in order to avoid unwanted Julia olefination with benzaldehyde. For benzothiazolyl sulfones, LiHMDS proved to be the best base, while the use of potassium bases led to poor conversions and selectivities (Scheme 6). The same reaction conditions were applied with success to pyridyl sulfones, but the selectivity was improved in this case with KHMDS. Successful Julia olefination was performed on both series of benzylidene acetal-sulfones, employing $\mathrm{NaH}$ in THF for benzothiazolyl derivatives and KHMDS in dichloromethane for pyridyl compounds. 


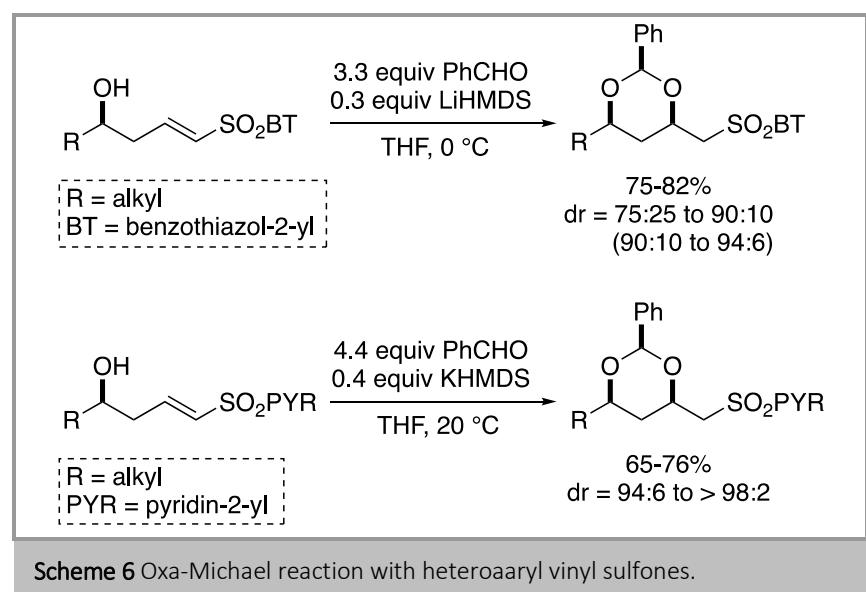

Another option for the synthesis of syn diols with a neighboring olefin is to install an aldehyde near the dioxane ring. For that purpose, substrates bearing a carbamate-substituted vinyl sulfone were prepared from the corresponding Hoppe homoaldol adducts ${ }^{13}$ (Scheme 7). These vinyl sulfones underwent conjugate addition in good yields and excellent selectivity. ${ }^{14}$ Reduction of the carbamate with $\mathrm{LiAlH}_{4}$ in THF at reflux, which occurred with concomitant elimination of benzenesulfinate, led to the corresponding aldehyde, but this aldehyde was subsequently reduced to the primary alcohol. Reoxidation of this alcohol with IBX furnished the desired aldehyde. This new methodology was successfully applied to the synthesis of the C16-C24 portion of Dolabelides.

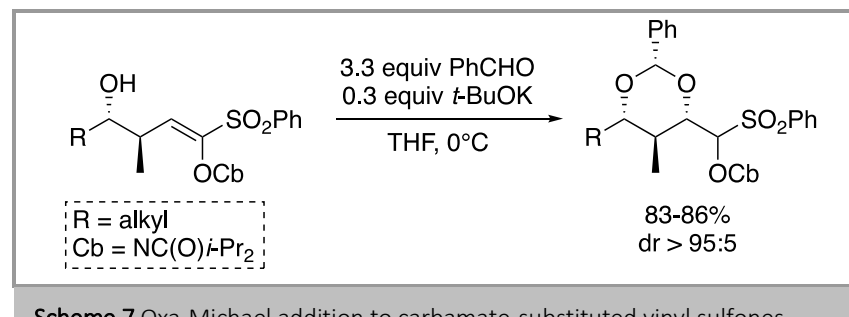

Scheme 7 Oxa-Michael addition to carbamate-substituted vinyl sulfones.

The sulfoxide group can serve both as Michael acceptor and as precursor to an aldehyde functional group. Prunet and GambaSánchez exploited this versatility by performing the oxa-Michael addition on vinyl sulfoxides (Scheme 8a), which led to the corresponding benzylidene acetals in good yields and selectivities. ${ }^{15}$ The configuration of the sulfoxide stereocenter has no bearing on the selectivity of the reaction, but the $E$ vinyl sulfoxides react faster than the $Z$ isomers. The scope of the reaction includes diverse alkyl groups for $\mathrm{R}_{1}$ (linear, branched, oxygenated) and the presence of a methyl group at the allylic position $\left(\mathrm{R}_{2}=\mathrm{Me}\right)$, although the selectivity is slightly lower when the methyl substituent is syn to the hydroxyl group, as it is the case for the conjugated ester substrates. The reaction was extended to tertiary alcohols (Scheme 8b). In this case, when the $\mathrm{R}$ group is similar in size to the methyl substituent, no selectivity is observed, as there is no intrinsic bias for the methyl group to occupy an axial position in the dioxane ring. The selectivity is excellent for $\mathrm{R}=t$-Bu but the conversion is poor. The yield is greatly improved when the reaction is conducted in ether. The sulfoxide products could easily be transformed into the corresponding aldehydes by Pummerer rearrangement. ${ }^{16}$
Further treatment of these aldehydes with CSA leads to unnatural ribofuranoses.

4.4 equiv $\mathrm{PhCHO}$
0.4 equiv $t$ - BuOK

Other aromatic aldehydes were employed in the oxa-Michael addition to conjugated esters. Since the benzylidene acetal requires harsh conditions for hydrolysis, it is not always suitable for the synthesis of complex polyketides. Evans and coworkers used $p$-anisaldehyde en route to Oasomycin $\mathrm{A}^{17}$ (Scheme 9a). In order to obtain a decent conversion, the reaction was performed in a non-polar solvent system ( $\mathrm{Et}_{2} \mathrm{O} /$ toluene).

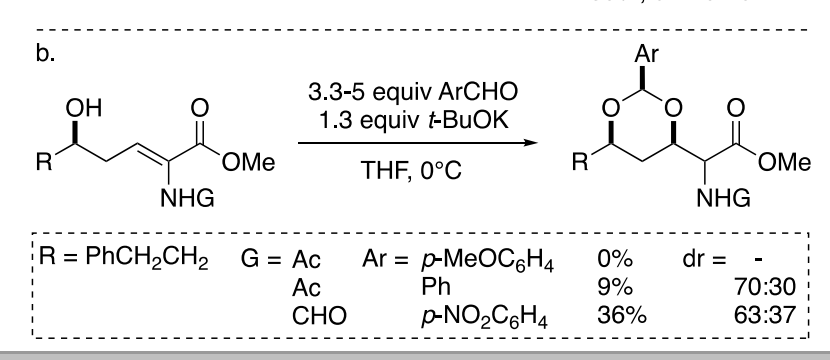

Scheme 9 Oxa-Michael addition to conjugated esters with diverse aromatic aldehydes.

The 1-amino-2,4-diol motif is commonly found in natural products. When Gamba-Sánchez and Miscione et al. treated conjugated esters with a protected $\alpha$-amino group with excess aromatic aldehyde and $t$-BuOK in order to synthesize such a motif, 18 there was a definite trend: electron-poor aromatic aldehydes gave better conversion (Scheme 9b). ${ }^{19}$ The reaction was then optimized by the same authors who found out that LiHMDS was the best base for this transformation (Scheme 10a). Conversion was highly dependent on the amino protecting group. With carbamates and trifluoroacetamido group, the yields were around $30 \%$, while with amides, yields up to $79 \%$ were obtained. In all cases, the 2,3- syn selectivity was modest. 


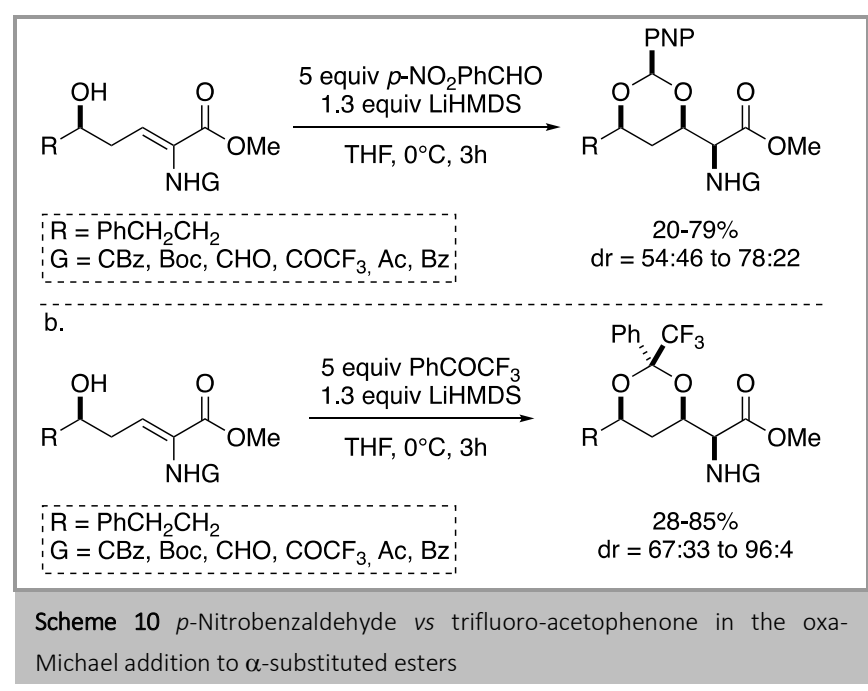

When trifluoroacetophenone was used as an aldehyde surrogate in the oxa-Michael addition on the same substrates (Scheme $10 \mathrm{~b})$, similar yields were observed with all substrates, but the 2,3- syn selectivity was better than 90:10 in most cases.

The scope of the oxa-Michael addition with trifluoroacetophenone was explored by Gamba-Sánchez and Prunet et $a l^{20}$ The results were comparable to the corresponding reactions with benzaldehyde for a range of conjugated esters (Scheme 11a), but also for conjugated Weinreb amides and vinyl sulfones (not shown).

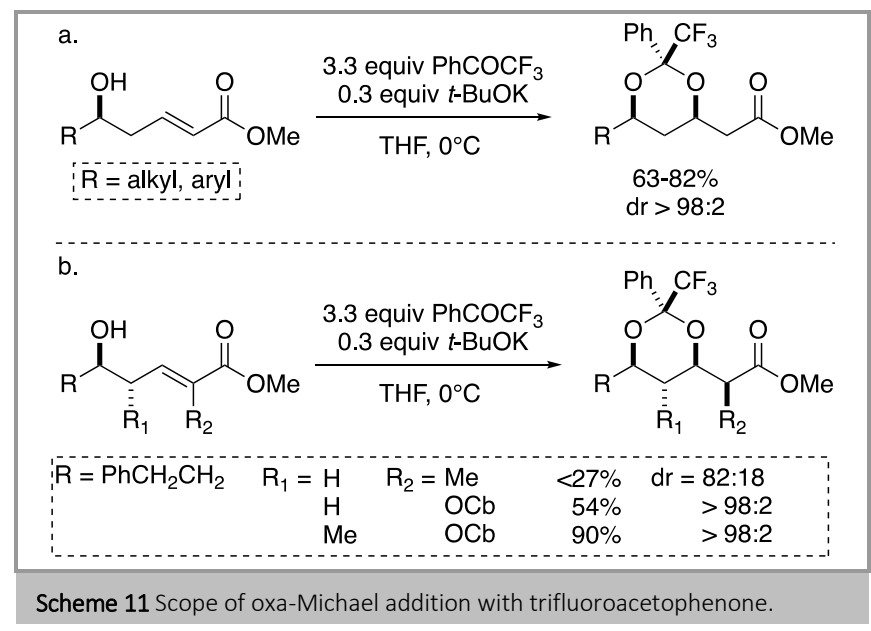

The real advantage of trifluoroacetophenone is that conversion is much higher with $\alpha$-substituted conjugated esters. For example, the oxa-Michael addition to the $\alpha$-acetamido ester only proceeds in 9\% yield with benzaldehyde (Scheme 9b), while the same reaction on the formamido derivative with trifluoroacetophenone leads to the desired product in $85 \%$ yield. Substrates with simple alkyl substituents $\left(\mathrm{R}_{2}=\mathrm{Me}\right.$, Scheme 11b) gave poor conversion (no reaction was observed with benzdehyde), but the carbamate derivatives led to dioxane products in good to excellent yields. It is worth noting that the reaction performed on the latter carbamate $\left(R_{1}=M e\right)$ with benzaldehyde proceded in only $35 \%$ yield with a 67:33 2,3-syn selectivity (vs $\quad 90 \% \quad$ yield, $98: 2 \quad$ selectivity with trifluoroacetophenone).
Trifluoroacetophenone is also very effective for the synthesis of protected 1,2-diols from quinol derivatives. ${ }^{21}$ Furthermore, the reaction was rendered enantioselective when (DHQ) ${ }_{2}$ PHAL was used as the catalyst.

Another option to introduce a new stereocenter from an achiral allylic alcohol was reported by Watanabe and coworkers. ${ }^{22}$ When treated with the ketone developed by Shi et al. for asymmetric epoxidation, ${ }^{23}$ in the presence of an ammonium hydroxide base, the primary alcohol shown in Scheme 12 underwent smooth conjugate addition to give two diastereomers of the ketal product in good yield and excellent selectivity. These two diastereomers differ at both newly created stereocenters. The reaction is thought to be under kinetic control, as longer reaction times, increased amounts of base or higher temperatures lead to reduced diastereoselectivity. The alcohol attacks preferentially the convex face of the ketone, and the conjugate addition then proceeds via the favored chair transition state $\mathbf{A}$. The minor diastereomer stems from attack to the ketone concave face, resulting in transition state B. Conjugated esters or Weinreb amides are unreactive under these conditions, and reaction with allylic alcohols is not diastereoselective for the formation of protected 1,2-diols.

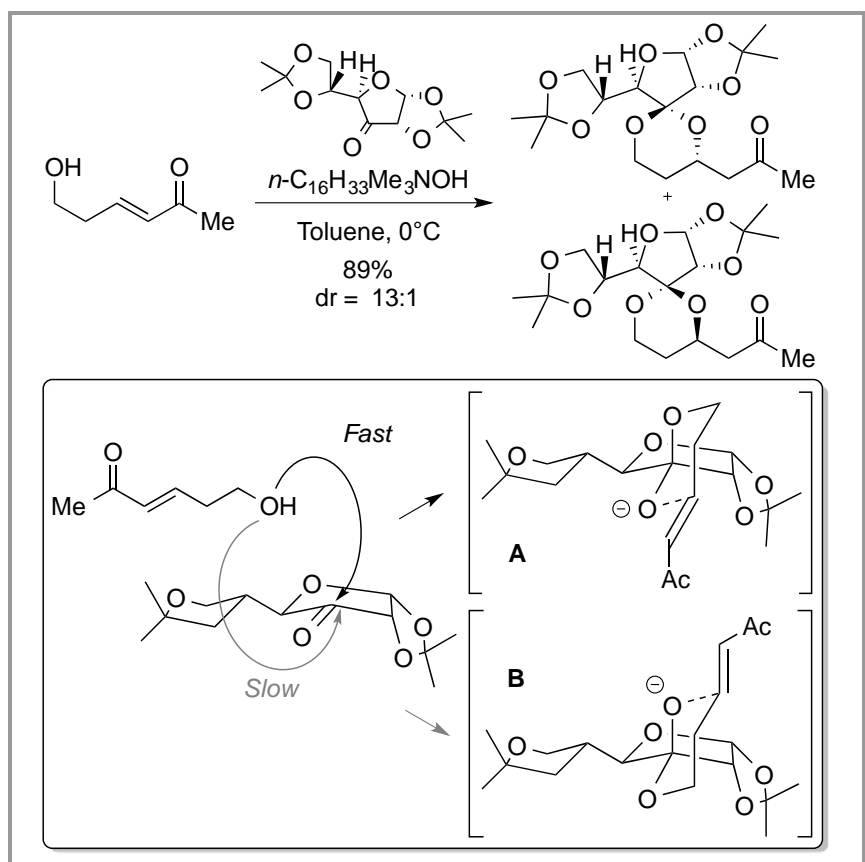

Scheme 12 Diasteroselective formation of acetals by oxa-Michael reaction with a chiral ketone.

\section{Organocatalysis}

Jefford and coworkers reported the acid-catalyzed oxa-Michael reaction on quinol substrates with aromatic aldehydes. ${ }^{24}$ More recently, Rovis et al. developed an enantioselective version of this reaction with aliphatic aldehydes and a BINOL-derived chiral phosphoric acid catalyst. ${ }^{25}$ This reaction proceeds under kinetic control. More recently, Asano and Matsubara et al. applied very similar reaction conditions to conjugated ketones with a primary homoallylic alcohol to obtain the corresponding 
acetals (Scheme 13). ${ }^{26}$ Excellent ees are obtained with aromatic ketones ( $\geq 89 \%$ ), while the selectivity of the reaction is only moderate with aliphatic and vinylic ketones (63-78\% ee). No reaction was observed with benzaldehyde, nor with conjugated esters as the Michael acceptors; however the benzylidene acetal ester products could be easily obtained from the corresponding ketones by Baeyer-Villiger oxidation without loss of enantiopurity.

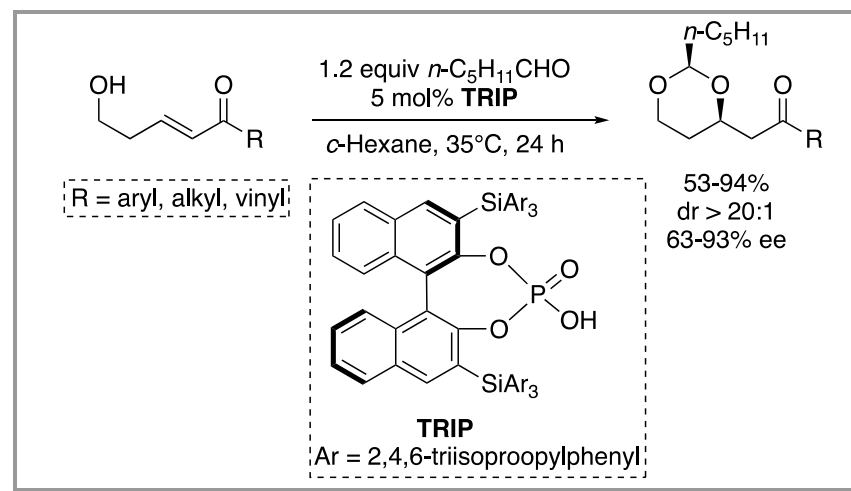

Scheme 13 Enantioselective formation of acetals by oxa-Michael reaction with a chiral phosphoric acid catalyst.

Falck and coworkers designed an ingenious reaction where the relay for the conjugate addition is not an aldehyde or a ketone, but a boronic acid (Scheme 14).27 The corresponding boronate esters are easily cleaved under oxidative conditions. They first optimized the reaction on the corresponding allylic alcohols. Inorganic or non-nucleophilic bases did not catalyze the reaction, but the five-membered ring boronate was obtained in $48 \%$ yield with DABCO and $86 \%$ yield with diisopropylamine. They then developed the asymmetric version of this reaction with a push-pull quinine-urea catalyst originally conceived by Soós et al. for the conjugate addition of nitromethane to enones. ${ }^{28}$ They showed that the urea moiety is essential for activity. Homoallylic alcohols were less reactive substrates, and the aliphatic enones required 3,4,5-trimethoxyboronic acid for good conversion, as it increased the nucleophilicity of the boronate intermediate. The opposite enantiomers of the diols could be obtained with the corresponding quinidine-urea catalyst. The same quinidine-urea catalyst was later employed by Asano and Matsubara et al. for the synthesis of fivemembered ring acetals from conjugated enones ${ }^{29}$ and thioesters ${ }^{30}$ with cyclohexanecarboxaldehyde.

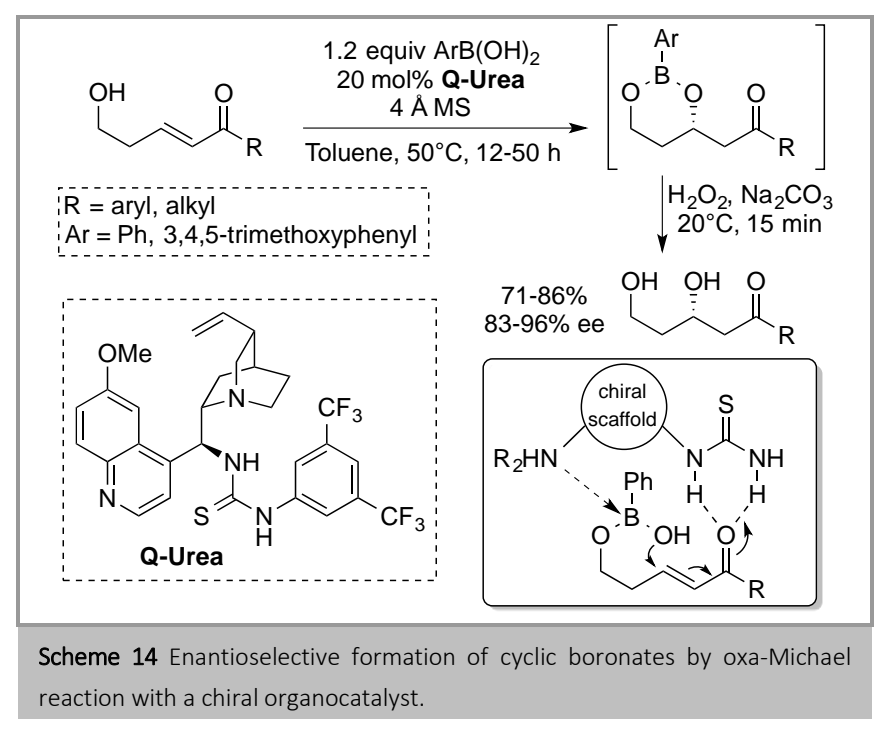

\section{Activation with non-metallic electrophiles}

Bartlett and Jernstedt published the first synthesis of protected 1,3-diols using activation with electrophiles. ${ }^{31}$ Their work was based on the formation of a iodonium ion and its reaction with a pending oxygen nucleophile from a phosphate; the selectivity is reached by an apparent equilibrium between the cyclic intermediate and the acyclic olefin (Scheme 15a).

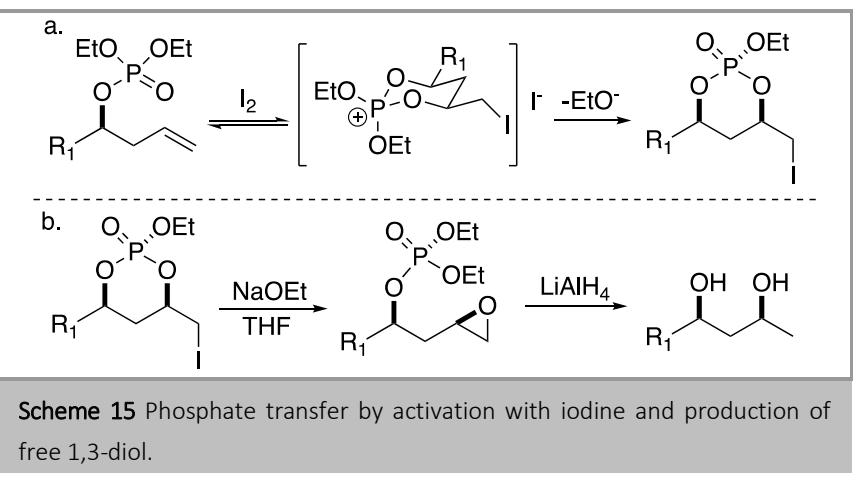

These authors were able to obtain epoxides under basic treatment and subsequent reduction afforded the free diols (Scheme 15b). ${ }^{32}$ The reaction is compatible with several alkyl groups and the selectivity is excellent; the biggest issue of this method is the reaction time, typically 2 days.

Latter the same authors extended their method to the use of carbonates. ${ }^{32}$ Unfortunately the selectivity was lower and the reaction scope somehow limited; however, the versatility of the carbonates makes the method interesting, in consequence Cardillo et al. $^{33}$ described the reaction using homoallylic alcohols as substrates and iodine as the activator. The reaction is performed using $n$-BuLi as a base, and the anion formed by deprotonation of the alcohol reacts with $\mathrm{CO}_{2}$ producing in situ the intermediate carbonate; the addition of iodine activates the double bound forming the iodonium ion, which is electrophilic enough to react with the negative oxygen (Scheme 16a). Selectivities were superior to $95 \%$ favouring the cis isomer, and yields were also good. The free diol is obtained under basic conditions; sadly the use of $\mathrm{CO}_{2}$ gas is a serious drawback of this 
method. Improvements ${ }^{34}$ and applications in the synthesis of polyols ${ }^{35}$ have been described despite the use of gaseous reagents. The use of tert-butyl carbonates and iodine monobromide (IBr) at low temperature improved the selectivity and avoid the use of $\mathrm{CO}_{2}$, this was published by Smith III and Duan in $1993^{36}$ (Scheme 16b). Examples of applications in natural products synthesis are the total synthesis of peloruside published by Taylor ${ }^{37}$ and the total synthesis of polyrhacitide $\mathrm{A}$ and epi-cryptocaryolone by Yadav et al.; ${ }^{38}$ in both cases the method with tert-butyl carbonates was employed with slight modifications.

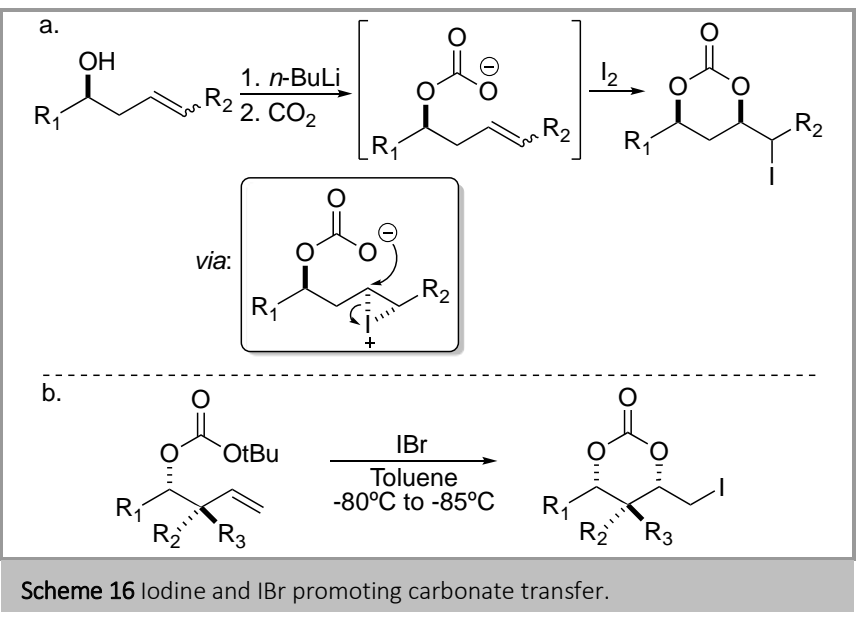

Nakada and coworkers described a similar approach ${ }^{39}$ using sulfates instead of phosphates or carbonates; the reaction is performed with $\mathrm{SO}_{3}$-Py and homoallylic alcohols, activation of the double bond is achieved with iodine or NBS, but the best results are obtained with $\mathrm{I}_{2}$ and $\mathrm{CH}_{3} \mathrm{CN}$ as the solvent. The selectivity is excellent in most of the cases and the reaction conditions avoid the use of gases (Scheme 17).

$$
\text { Scheme } 17 \text { Direct sulfate transfer using a masked } \mathrm{SO}_{3} \text { equivalent. }
$$

An enantioselective version of this reaction was recently published using an organocatalytic approach. ${ }^{40}$ The method is useful to create quaternary centers and uses $\mathrm{CO}_{2}$ as the carbonate source and NIS as the activator of the double bond (Scheme 18). Authors made an extensive test of catalysts and conditions establishing that $\mathrm{Br} ø$ nsted acid/base combination is essential to promote the reaction. No details about the mechanism were provided, but probably the catalyst is involved in a hydrogen bond network in the selectivity-determining step.

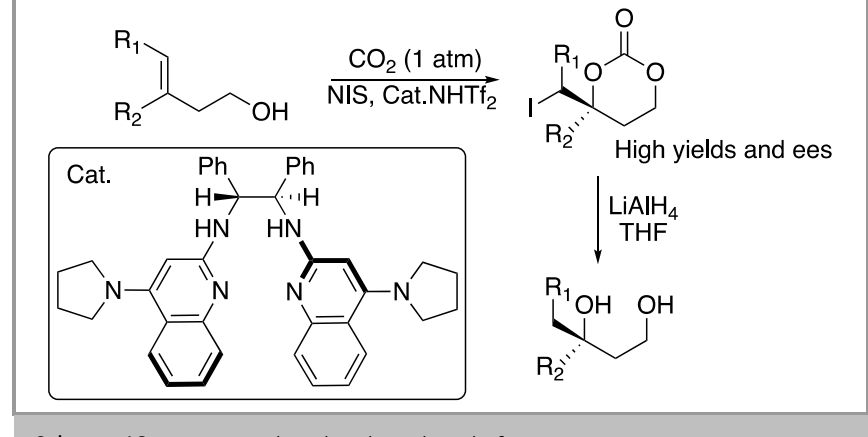

Scheme 18 Organocatalyzed carbon dioxide fixation

A related methodology is the ether transfer; ${ }^{41}$ the main idea is the use of simple acetals instead of carbonates, phosphates, sulfates, etc. In fact after the activation of the double bond with a electrophilic reagent, an oxygen from an ether can react as a nucleophile generating a oxonium ion intermediate, which can be trapped by a variety of nucleophiles. This method was developed by Taylor et al. ${ }^{42}$ and has been applied successfully to the synthesis of 2,4,6-trisubstituted tetrahydropyrans ${ }^{43}$ and 2,6disubstituted-3,4-dihydropyrans. ${ }^{44}$ The main idea and applications are illustrated in Scheme 19.

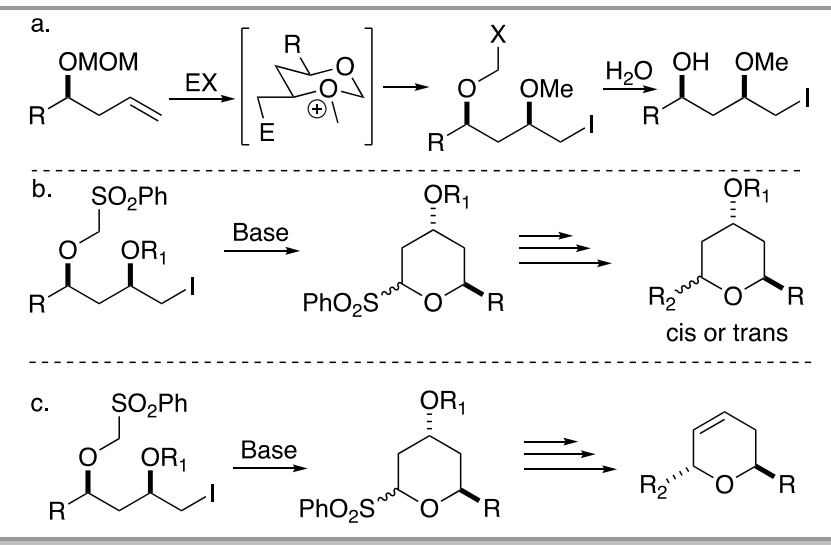

Scheme 19 Ether transfer in the synthesis of 1,3-diols and hydropyrans.

The use of methoxymethyl ethers as substrates is very useful in the synthesis of natural products bearing one methyl ether as part of the 1,3-dioxygenated system; on the other hand, the use of benzyloxymethyl ether provides simple access to the free diol by subsequent cleavage of the benzyl ether. However, depending on the reaction conditions, competitive benzyl cleavage is observed providing the diol protected as a methylidene acetal (Scheme 20).

Scheme 20 benzyloxymethyl ether transfer, production of fully protected 1,3-

The examples presented in this section are based on the activation of double bonds by non-metallic reagents. The 
activation with transition metal derivatives in similar processes is discussed below.

\section{Activation with transition metal derivatives}

The base catalyzed synthesis of 1,3-diols has shown a tremendous range of applications in recent years as shown in the first section of this review article; however, the acidic version is still a challenge and very few reports are described in literature. Between them the activation with metals is an interesting approach that can be used even with electron-rich olefins. In fact, if the metal coordinates or reacts with a $\mathrm{C}=\mathrm{C}$ double bond, a new electrophilic center is created, and the addition of $O$-nucleophiles is feasible. Overman and Campbell published the pioneering work using this strategy in 1974.45 Their approach was based on the use of acetals obtained by the reaction of cyclic allylic alcohols and chloral, that they then reacted with mercury salts in order to obtain mainly 1,3dioxolanes, which can be seen as protected 1,2-diols. The use of homoallyllic alcohols did not produced pure 1,3-dioxanes but mixtures with 1,3-dioxepanes. Authors explained the formation of the seven-membered ring as a major isomer by kinetic factors. Ten years later Giese et al. ${ }^{46}$ extended the method to the use of acyclic alcohols. Those methodologies have several drawbacks; they are limited by the use of chloral and aliphatic aldehydes, which makes the acetal hydrolysis and their application in total synthesis difficult; and the major product is always the 1,2-diol.

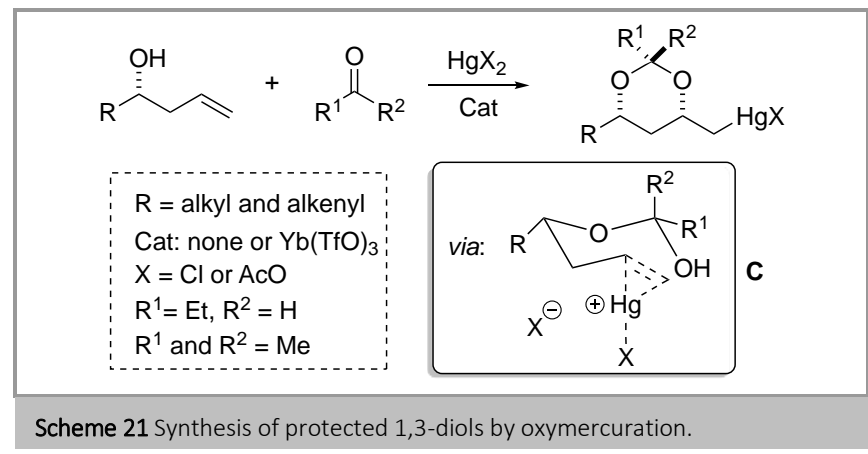

Leighton and coworkers improved the method and made it applicable to synthesize protected 1,3-diols by tandem additionoxymercuration reaction ${ }^{47}$ (Scheme 21).

The first Leighton approach used propionaldehyde and homoallylic alcohols. The reaction between them (induced by mercury salts) produced small quantities of hemiacetal in equilibrium with the reagents. However, the free $\mathrm{OH}$ group of the hemiacetal is nucleophilic enough to react with the mercurinium ion generated between the double bond and the mercury reagent; this last step is irreversible as suggested by the authors. They explained the stereoselectivity by the rapid cyclization of intermediate $\mathbf{C}$; its formation is reversible and governed by the steric hindrance of substituents in pseudo equatorial positions, but the cyclization is the rate determining step and faster in the more stable intermediate. $47 \mathrm{a}$

Later the same year Leighton introduced the use of $\mathrm{Yb}(\mathrm{OTf})_{3}$ as catalyst for this reaction. ${ }^{47 b}$ The use of this reagent allowed not only a significantly increase of the reaction rate but also extended the reaction to the use of acetone, which is less electrophilic than aliphatic aldehydes but leads to easily hydrolyzed acetonides. On the other hand, they demonstrated that in this case the reaction is under thermodynamic control and the stereoselectivity is achieved by equilibration of the intermediates; thus, the catalyzed version of this reaction is influenced by ytterbium in the activation of carbonyl compound and in the oxymercuration step.

Oxymercuration in the synthesis of 1,3-diols was also used by Cossy and Meyer using cyclopropanes. ${ }^{48}$ They obtained promising results but the method still lacks generality and the products were obtained in low yields.

The use of other metals remained unexplored until 2011 when P. A. Evans and coworkers ${ }^{49}$ described the first hemiacetal formation/oxa-Michael addition sequence using aldehydes and ketones as Michael acceptors. The reaction is promoted by $\mathrm{Bi}\left(\mathrm{NO}_{3}\right)_{3}$ and produced syn 1,3-diols with excellent selectivities. The reaction is performed either with free or protected alcohols as described in Scheme 22. The most important advantage of this method is that it can be applied to ketones and aldehydes as Michael acceptor which is not the case in the base catalyzed version of this reaction. The main disadvantage is that it is limited to the use of aliphatic aldehydes, and even if their acetals can be hydrolyzed to produce the free diols, the conditions needed are harsher than with aromatic acetals. The catalytic role of bismuth was proved carrying out the reaction with nitric acid with significantly lower yields. As an extension, Chen and coworkers ${ }^{50}$ applied this method to the synthesis of pitavastatin calcium. They tried other bismuth sources and solvents, but again the nitrate and dichloromethane showed the best results.

Scheme 22 Bismuth catalyzed addition/oxa-Michael sequence using
$\mathrm{PWG}=\mathrm{CHO}, \mathrm{COMe}$
aldehydes and ketones as Michael acceptors.

The use of additives like $\mathrm{LiNO}_{3}$, LiOTf, $\mathrm{NaClO}_{4}$, and $\mathrm{LiClO}_{4}$ allowed the use of other bismuth sources and aromatic aldehydes as the electrophilic partner as described by Hayashi et $a .^{51}$ The best results were obtained using stoichiometric amounts of $\mathrm{NaClO}_{4}$ and $10 \% \mathrm{~mol}$ of $\mathrm{Bi}(\mathrm{OTf})_{3}$. These authors showed the synthetic utility of the additives in the synthesis of Diospongin C and Yashabushidiol A.

Very interesting approaches using activation of double bonds with transition metals based on Tsuji-Trost type reactions have been reported. In fact a hemiacetal (generated in situ) is a suitable nucleophile in Tsuji-Trost allylic transpositions; in consequence many combinations of starting materials are reasonable to construct 1,3-diols.

Menche and Wang ${ }^{52}$ reported the first approach using one of those strategies. They used a palladium catalyst and acetaldehyde to generate the hemiacetal, and carbonates as leaving groups (Scheme 23). 


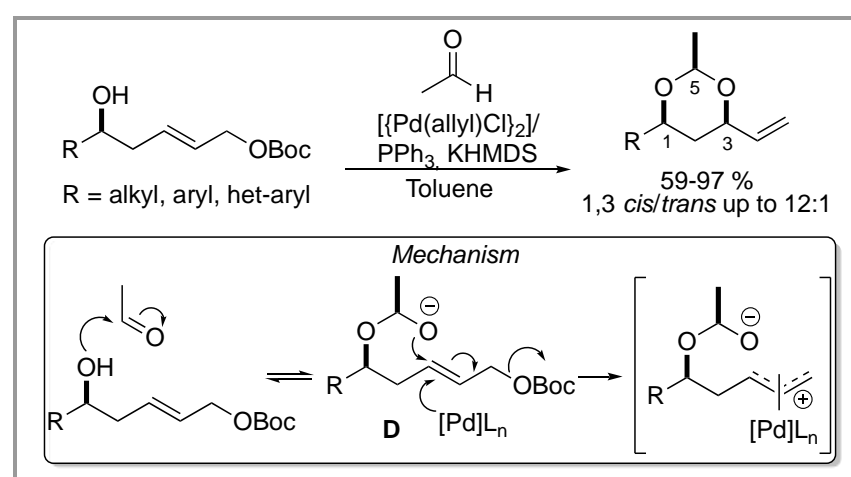

Scheme 23 Palladium catalyzed intramolecular allylic substitution.

After optimization of the reaction conditions, different substrates were used successfully; good yields and selectivities were obtained in all cases showing a very good substrate scope. The selectivity is attributed to a three-step mechanism. The first step is the reversible generation of the hemiacetal; two diastereomers will be formed but the equilibration to the more stable transition state is determinant for a good selectivity. The second step is the formation of the $\pi$-allyl complex, followed by the intramolecular allylic substitution as the third step. The last two steps only proceed with the more stable isomer of the intermediate $\mathbf{D}$.

A similar approach was used some years later by Aponik and coworkers, ${ }^{53}$ with some differences. First the method is applicable to $E$ alkenes with $\mathrm{Bi}^{3+}$ catalysts and to $Z$ alkenes with $\mathrm{Au}^{+}$catalyst, and second the leaving group is water. In this case authors suggest a tandem hemiacetalization/dehydrative cyclization process; the mechanism presumably follows a reversible dehydrative cyclization, since removing water afford the best results in terms of yield and selectivity. It has to be noted that the method can be applied to the synthesis of 1,2diols as well (Scheme 24).

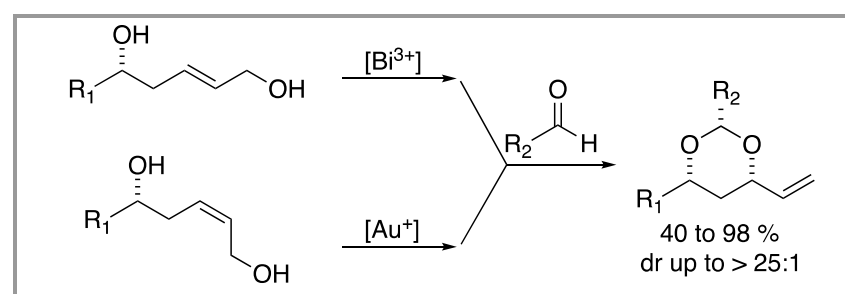

Scheme 24 Gold and bismuth catalyzed synthesis of protected 1,3-diols.

In all former approaches using metallic activation, carbonyl compounds -aldehydes and more recently ketones- have been used to produce protected 1,3-diols as acetals. The use of carbonates even if less common is very interesting since the products can be easily converted into the corresponding free diols under basic conditions. This served as inspiration to Cossy and coworkers ${ }^{54}$ to develop an alternative method that can be applied to the synthesis of 1,2- and 1,3-diols. Their method is based on the formation of an allylic cation by the elimination of an acetate group (Scheme 25). This process is induced by metals like iron and indium. The method is simple to carry out, it can be performed with hydrated iron chloride which is easy to handle, and it can be applied to the synthesis of 1,2-diols; unfortunately the diastereoselectivity is poor compared with other existing methods, and the substrate scope is limited to the use of phenyl allylic cations as intermediates. These authors suggested a kinetic control after NMR studies; however, they recognized that further mechanistic studies are still needed. On the other hand, they applied successfully their methodology to the synthesis of alpinikatin.

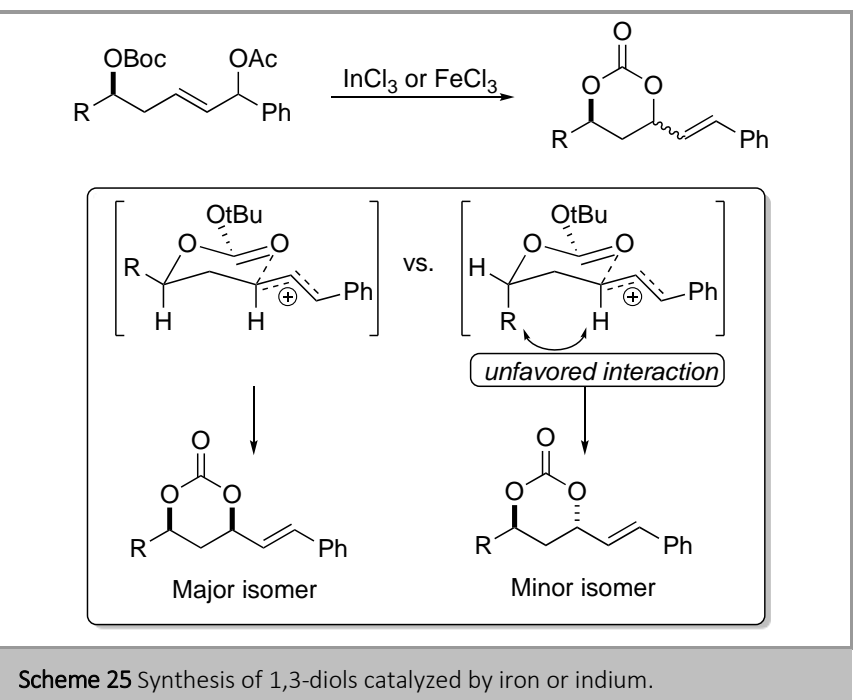

The former approaches are based on the formation of a sixmembered rings that control the stereochemistry by equilibration of the transition state or the final product. In consequence, an allylic transposition without the concomitant formation of a cyclic compound should derive in a nonstereoselective reaction. Zakarian and coworkers ${ }^{55}$ front faced this problem using rhenium as catalyst and alcohols as the staring materials (Scheme 26).

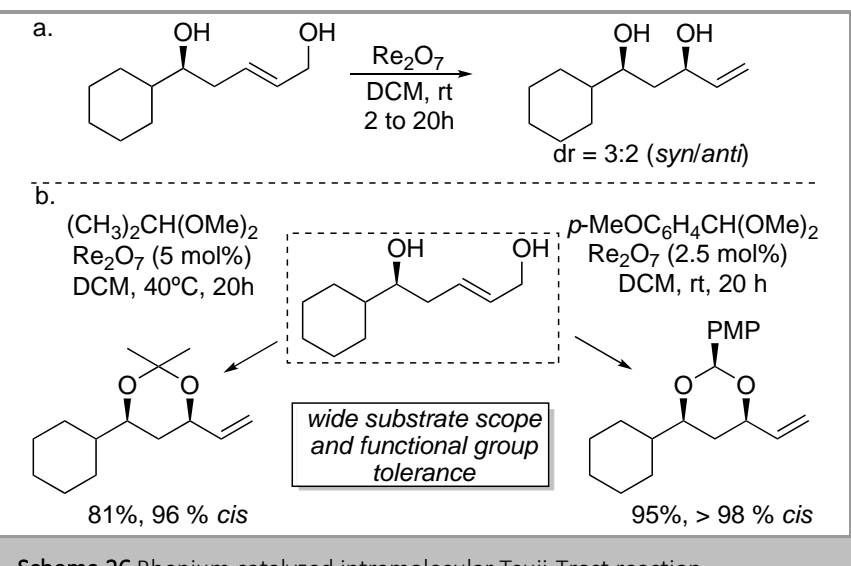

Scheme 26 Rhenium catalyzed intramolecular Tsuji-Trost reaction.

These authors showed that the allylic transposition generates the product with poor selectivity (Scheme 26a). However, trapping the 1,3-diol as an aromatic acetal or ketal allows the equilibration of the intermediate to the thermodynamically more stable cis isomer (Scheme 26b); that means the rhenium performs a dual role, catalyst for allylic transposition and as activator of the carbonyl compound.

Complementary studies carried out by Kitamura and coworkers $^{56}$ used primary alcohols and a chiral ruthenium catalyst; using this combination these authors were able to synthesize 1,3-diols creating a new chiral center with good enantiomeric ratio and in gram scale (Scheme 27). This 
methodology was also applied to the synthesis of 1,2-diols, 1,2aminoalcohols and 1,3-aminoalcohols with similar results.

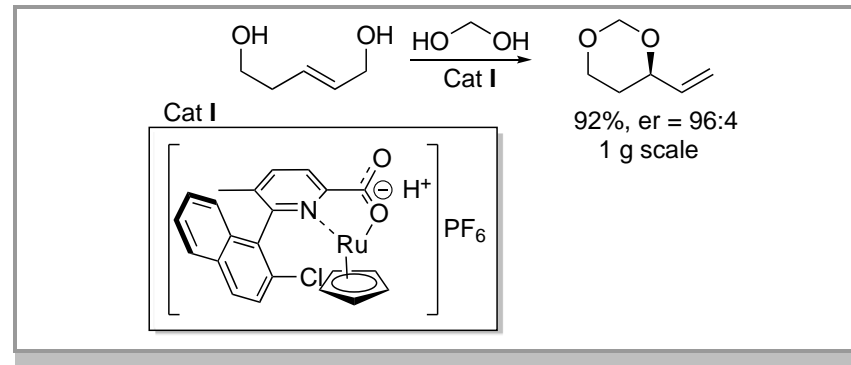

Scheme 27 Asymmetric Tsuji-Trost reaction in the synthesis of 1,3-diols.

Allylic cations, as equivalents of electron-poor double bonds, can also be obtained directly from the interaction of some metals with allenes; this was recently described by Breit and Spreider ${ }^{57}$ using a palladium catalyst and formaldehyde (Scheme 28). The method is water compatible since it uses aqueous formalin as source of formaldehyde, yields and selectivities are excellent, and also the reaction has a wide substrate scope. These authors demonstrated that the reaction is under thermodynamic control and the selectivity is achieved by the equilibration to the more stable dioxane with all the substituents in equatorial positions. The application to the synthesis of pitavastatin calcium and rosuvastatin calcium demonstrated the versatility of this method.

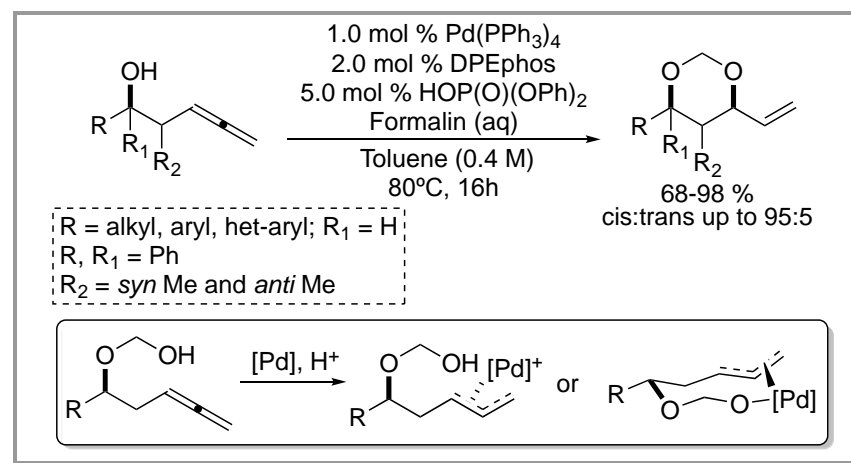

Scheme 28 Synthesis of syn 1,3-doils using allenes and paladum catalyst.

An amazing copper-catalyzed oxy-alkenylation was described by Gaunt and Holt. ${ }^{58}$ The combination of copper salts with aryl alkenyliodonium reagents provides highly electrophilic species, which can react with double bonds, activating the addition of oxygen nucleophiles. The reaction is performed with carbamates and under hydrolytic conditions the 1,3-diols are obtained as carbonates. These authors suggested a $\mathrm{Cu}(\mathrm{III})$ intermediate as the responsible for the activation of the double bond, and the addition of the pending oxygen nucleophile generates the 1,3-diol system that undergoes a reductive elimination and hydrolysis to produce the desired carbonates (Scheme 29a). The scope is very good and additional chiral centers can be introduced by controlling the stereochemistry of the reacting double bond (Schemes $29 \mathrm{~b}$ and 29c).

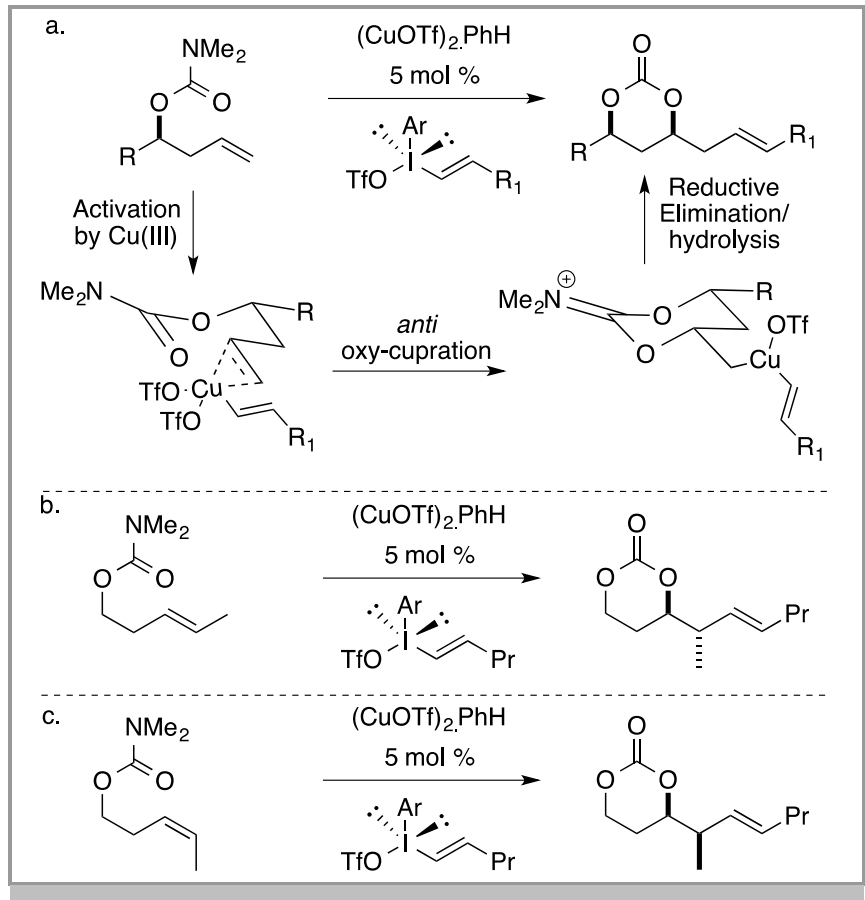

Scheme 29 Copper-catalyzed oxy-alkenylation.

\section{Conclusion}

The 1,3-diol motif is present in a large variety of natural products and in consequence its synthesis has been the focus of many researchers in the last 30 years. Most methods for the synthesis of this important scaffold, not involving carboncarbon bond formation or carbonyl reduction, are based on the nucleophilic addition of oxygen to electro-poor double bonds or similar functions. We have presented in this review a selected set of examples showing the variety of strategies and substrates used in synthesis of 1,3-diols, principally focusing on the synthesis of syn derivatives. The methods are generally Michael additions in basic or acidic medium, but the activation of homoallylic alcohols with electrophilic reagents has also been described with success. We hope this overview will help researchers as inspiration in their future work and that they will found it useful in the comprehension of some commonly used methods.

\section{Funding Information}

Financial support was provided by Fondo de Investigaciones de la Facultad de Ciencias de la Universidad de los Andes, convocatoria 20182019 para la Financiación de Programas de Investigación "use of threonine as chiral auxiliary"

\section{Acknowledgment}

D.G.-S. acknowledges the Chemistry Department of Universidad de los Andes and J.P. the University of Glasgow for logistical support.

\section{References}

(1) Bode, S. E.; Wolberg, M.; Müller, M. Synthesis 2006, 557.

(2) Kumar, P.; Tripathi, D.; Sharma, B. M.; Dwivedi, N. Org. Biomol. Chem. 2017, 15, 733.

(3) For a recent review of the application of the oxa-Michael reaction to the synthesis of natural products, see Hu, J.; Bian, M.; Ding, $\mathrm{H}$. Tetrahedron Lett. 2016, 57, 5519. 
(4) Evans, D. A.; Gauchet-Prunet, J. A. J. Org. Chem. 1993, 58, 2446.

(5) Prunet, J. PhD Thesis, Harvard University, 1993.

(6) (a) Hung, D. T.; Nerenberg, J. B.; Schreiber, S. L. J. Am. Chem. Soc. 1996, 118, 11054. (b) Dineen, T. A.; Roush, W. R. Org. Lett. 2004, 6 , 2043. (c) Hunter, T. J.; O'Doherty, G. A. Org. Lett. 2001, 3, 2777. (d) Vincent, A.; Prunet, J. Synlett 2006, 2269. (e) de Lemos, E.; Porée, F.H.; Bourin, A.; Barbion, J.; Agouridas, E.; Lannou, M.-I.; Commerçon, A.; Betzer, J.-F.; Pancrazi, A.; Ardisson, J. Chem. Eur. J. 2008, 14 11092. (f) Palimkar, S. S.; Uenishi, J. i. Org. Lett. 2010, 12, 4160. (g) Albury, A. M. M.; Jennings, M. P. J. Org. Chem. 2012, 77, 6929. (h) Bates, R. W.; Lek, T. G. Synthesis 2014, 46, 1731. (i) Hunter, T. J.; Wang, Y.; Zheng, J.; O’Doherty, G. A. Synthesis 2016, 48, 1700.

(7) Grimaud, L.; Rotulo, D.; Ros-Perez, R.; Guitry-Azam, L.; Prunet, J. Tetrahedron Lett. 2002, 43, 7477.

(8) Rotulo-Sims, D.; Grimaud, L.; Prunet, J. C. R. Chim. 2004, 7, 941.

(9) Rotulo-Sims, D.; Prunet, J. Org. Lett. 2007, 9, 4147.

(10) Aouzal, R.; Prunet, J. Org. Biomol. Chem. 2009, 7, 3594.

(11) (a) Baudin, J. B.; Hareau, G.; Julia, S. A.; Ruel, O. Tetrahedron Lett. 1991, 32, 1175. (b) Baudin, J. B.; Hareau, G.; Julia, S. A.; Ruel, O. Bull. Soc. Chim. Fr 1993, 130, 336. (c) Baudin, J. B.; Hareau, G.; Julia, S. A.; Ruel, O. Bull. Soc. Chim. Fr 1993, 130, 856. (d) Charette, A. B.; Berthelette, C.; St-Martin, D. Tetrahedron Lett. 2001, 42, 5149.

(12) Oriez, R.; Prunet, J. Tetrahedron Lett. 2010, 51, 256.

(13) Inga, H.; Dieter, H.; Christian, W.; Ernst, E.; Regine, H. Angew. Chem. Int. Ed. 1989, 28, 67.

(14) Grimaud, L.; de Mesmay, R.; Prunet, J. Org. Lett. 2002, 4, 419.

(15) Gamba-Sanchez, D.; Prunet, J. J. Org. Chem. 2010, 75, 3129.

(16) Gamba-Sanchez, D.; Garzon-Posse, F. In Molecular Rearrangements in Organic Synthesis; Rojas, C., Ed.; John Wiley \& Sons: 2015, p 661.

(17) A., E. D.; Pavel, N.; J., R. D.; J., M. K. Angew. Chem. Int. Ed. 2007, 46, 541.

(18) Becerra-Figueroa, L.; Movilla, S.; Prunet, J.; Miscione, G. P.; GambaSanchez, D. Org. Biomol. Chem. 2018, 16, 1277.

(19) Electron-poor aromatic aldehydes have also been employed with quinol derivatives for the synthesis of protected 1,2-diols, see: Redondo, M. C.; Ribagorda, M.; Carreño, M. C. Org. Lett. 2010, 12, 568.

(20) Becerra-Figueroa, L.; Brun, E.; Mathieson, M.; Farrugia, L. J.; Wilson, C.; Prunet, J.; Gamba-Sanchez, D. Org. Biomol. Chem. 2017, 15, 301.

(21) Li, F.; Wang, J.; Xu, M.; Zhao, X.; Zhou, X.; Zhao, W.-X.; Liu, L. Org. Biomol. Chem. 2016, 14, 3981.

(22) Hidenori, W.; Kaori, M.; Daisuke, I.; Haruki, N.; Takeshi, K. Chirality 2001, 13, 379.

(23) Tu, Y.; Wang, Z.-X.; Frohn, M.; He, M.; Yu, H.; Tang, Y.; Shi, Y. J. Org. Chem. 1998, 63, 8475.

(24) (a) Jefford, C. W.; Rossier, J.-C.; Kohmoto, S.; Boukouvalas, J. Synthesis 1985, 1985, 29. For the formation of trioxanes from hydroperoxides, see: (b) Jefford, C. W.; Jaggi, D.; Boukouvalas, J.; Kohmoto, S. J. Am. Chem. Soc. 1983, 105, 6497.

(25) (a) Rubush, D. M.; Rovis, T. Synlett 2014, 25, 713. For the asymmetric formation of trioxanes from hydroperoxides, see: (b) Rubush, D. M.; Morges, M. A.; Rose, B. J.; Thamm, D. H.; Rovis, T. J. Am. Chem. Soc. 2012, 134, 13554.
(26) Matsumoto, A.; Asano, K.; Matsubara, S. Chem. Commun. 2015, 51 11693.

(27) Li, D. R.; Murugan, A.; Falck, J. R. J. Am. Chem. Soc. 2008, 130, 46.

(28) Vakulya, B.; Varga, S.; Csámpai, A.; Soós, T. Org. Lett. 2005, 7, 1967.

(29) Asano, K.; Matsubara, S. Org. Lett. 2012, 14, 1620.

(30) Okamura, T.; Asano, K.; Matsubara, S. Chem. Commun. 2012, 48, 5076.

(31) Bartlett, P. A.; Jernstedt, K. K. J. Am. Chem. Soc. 1977, 99, 4829.

(32) Bartlett, P. A.; Meadows, J. D.; Brown, E. G.; Morimoto, A.; Jernstedt, K. K. J. Org. Chem. 1982, 47, 4013.

(33) Cardillo, G.; Orena, M.; Porzi, G.; Sandri, S. J. Chem. Soc., Chem. Commun. 1981, 465.

(34) Bongini, A.; Cardillo, G.; Orena, M.; Porzi, G.; Sandri, S. J. Org. Chem. 1982, 47, 4626.

(35) Lipshutz, B. H.; Kozlowski, J. A. J. Org. Chem. 1984, 49, 1147.

(36) Duan, J. J. W.; Smith, A. B. J. Org. Chem. 1993, 58, 3703.

(37) Taylor, R. E.; Jin, M. Org. Lett. 2003, 5, 4959.

(38) Mohapatra, D. K.; Bhimireddy, E.; Krishnarao, P. S.; Das, P. P.; Yadav, J. S. Org. Lett. 2011, 13, 744.

(39) Inoue, M.; Motomatsu, S.; Nakada, M. Synth. Commun. 2003, 33, 2857.

(40) Vara, B. A.; Struble, T. J.; Wang, W.; Dobish, M. C.; Johnston, J. N. J. Am. Chem. Soc. 2015, 137, 7302.

(41) Stefan, E.; Taylor, R. E. In Stereoselective Synthesis of Drugs and Natural Products; Andrushko, V., Andrushko, N., Eds.; Jhon Wiley \& Sons: 2013, p 1115.

(42) Liu, K.; Taylor, R. E.; Kartika, R. Org. Lett. 2006, 8, 5393.

(43) Kartika, R.; Taylor, R. E. Angew. Chem. Int. Ed. 2007, 46, 6874.

(44) Kartika, R.; Frein, J. D.; Taylor, R. E. J. Org. Chem. 2008, 73, 5592.

(45) Overman, L. E.; Campbell, C. B. J. Org. Chem. 1974, 39, 1474.

(46) Giese, B.; Bartmann, D. Tetrahedron Lett. 1985, 26, 1197.

(47) (a) Sarraf, S. T.; Leighton, J. L. Org. Lett. 2000, 2, 403. (b) Dreher, S. D.; Hornberger, K. R.; Sarraf, S. T.; Leighton, J. L. Org. Lett. 2000, 2, 3197.

(48) (a) Cossy, J.; Blanchard, N.; Meyer, C. Org. Lett. 2001, 3, 2567. (b) Meyer, C.; Blanchard, N.; Defosseux, M.; Cossy, J. Acc. Chem. Res. 2003, 36, 766.

(49) Evans, P. A.; Grisin, A.; Lawler, M. J. J. Am. Chem. Soc. 2012, 134, 2856.

(50) Xiong, F.; Wang, H.; Yan, L.; Xu, L.; Tao, Y.; Wu, Y.; Chen, F. Org. Biomol. Chem. 2015, 13, 9813.

(51) Hayashi, Y.; Saitoh, T.; Arase, H.; Kawauchi, G.; Takeda, N.; Shimasaki, Y.; Sato, I. Chem. Eur. J. 2018, 24, 4909.

(52) Wang, L.; Menche, D. Angew. Chem. Int. Ed. 2012, 51, 9425.

(53) Goodwin, J. A.; Ballesteros, C. F.; Aponick, A. Org. Lett. 2015, 17, 5574.

(54) Cornil, J.; Gonnard, L.; Guérinot, A.; Reymond, S.; Cossy, J. Eur. J. Org. Chem. 2014, 2014, 4958.

(55) Herrmann, A. T.; Saito, T.; Stivala, C. E.; Tom, J.; Zakarian, A. J. Am. Chem. Soc. 2010, 132, 5962.

(56) Tanaka, S.; Gunasekar, R.; Tanaka, T.; Iyoda, Y.; Suzuki, Y.; Kitamura, M. J. Org. Chem. 2017, 82, 9160.

(57) Spreider, P. A.; Breit, B. Org. Lett. 2018, 20, 3286.

(58) Holt, D.; Gaunt, M. J. Angew. Chem. Int. Ed. 2015, 54, 7857.

\section{Biosketches}

Diego Gamba-Sánchez was born in Bogotá, Colombia. He received his B.Sc. in Chemistry from the
Universidad Nacional de Colombia (Bogotá) in 2004 and his M.Sc. degree in Biomolecules and Organic
Synthesis from the Université de Poiters, France, in 2006; then, he moved to École Polytechnique,
Palaiseau where he worked under the guidance of Dr. Joëlle Prunet, and he received his Ph.D. in the field
of diastereoselective synthesis of 1,3-diols. Later, Diego joined the group of Prof. Thorsten Bach at
Technische Universität München, Germany, where he spent one year working on total synthesis of
natural products. Then, he moved back to Colombia and started his independent career at the
Universidad de los Andes, Bogotá, where he was promoted to Associate Professor in June 2014. The
research in his group focuses on the development of new synthetic strategies for amide synthesis, the
use of whole cells as biocatalysts, and the study of new methodologies for the synthesis of oxygenated
heterocycles using Pummerer reaction and intramolecular oxa-Michael additions.




Dr. Joëlle Prunet attended the Ecole Normale Supérieure (Paris) as an undergraduate and worked under
the supervision or Professor Marc Julia during her Master, then obtained her Ph.D. from Harvard
University with Professor David A. Evans in 1993. Afterwards she joined the CNRS as Chargée de
Recherche at the Ecole Polytechnique. In 2002 she was awarded the Bronze Medal from the CNRS, and
the following year she was promoted to Directrice de Recherche and obtained a teaching position at the
Ecole Polytechnique. In 2009, she was appointed to the University of Glasgow. Her research interests
include the total synthesis of bioactive natural products and the development of new methodologies in
organic chemistry. Recently, the she has been using cross and ring-closing metathesis reactions for the
post-polymerization functionalization of polymers of very diverse natures.

